\title{
A Web Services Signaling Approach over Optical Networks for SAN Applications
}

\author{
Omar Cherkaoui ${ }^{1}$, Nathalie Rico ${ }^{2}$, T. Dieu Linh Truong${ }^{1}$, \\ Halima Elbiaze ${ }^{1}$ and Viet Minh Nhat Vo ${ }^{1}$ \\ ${ }^{1}$ Université du Québec à Montréal, \\ C.P. 8888 succ. A., Montréal, H3C-3P8, Canada \\ 2 Université de Montréal
}

\begin{abstract}
Storage Area Network (SAN) promise both a single source of data and improved performance and availability. SAN are extended to a WAN infrastructure to offer easier sharing over multiple locations. This created a need for bandwidth which can be provided by the underlying optical transport network. In this paper, we propose a policybased lightpath signaling approach based on the OpenGrid architecture allowing SAN applications to dynamically establish and provision lightpaths over an optical network.
\end{abstract}

\section{SAN Management Architecture}

The storage industry is undergoing a revolution. The emerging technologies such as the SAN promise both a single source of data and improved performance and availability. A SAN is a means to allow multiple servers to have direct access to common storage devices or storage pool. Today, SAN are deployed over a LAN infrastructure. The increasing need to share information across organizations led companies to extend the SAN over WAN. IETF and other organizations proposed different solutions for realizing a SAN over the IP network. SAN applications need to have access to the required bandwidth provided by the underlying optical transport network to obtain adequate performance. We propose a lightpath signaling approach based on the OpenGrid architecture allowing SAN applications to establish and control lightpaths over an optical network. To address the admission control and scalability issues, we use a policy-based approach providing a flexible means to control the set-up of optical lightpaths.

VSAN (Virtual SAN) is like a virtual private network dedicated to exchange storage traffic. The network architecture for VSAN is composed of SANs interconnecting through the Internet WANs. To facilitate the management and control, a policy server in each SAN store the service policies. It is used to activate a service, to reserve the storage units and to configure the FC switches to build routes from the hosts to the storage units. A service provider who has available VSAN services advertises them on a web site. The web site may be located locally or on a common web server such as an UDDI (Universal Description, Discovery and Integration) database server. 
The proposed signaling approach is based on the Open Grid Services Architecture (OGSA) [2]. The architecture incorporates a policy framework to control the establishment of lightpaths on high-speed networks (see Figure 1). The OGSA integrates Grid technologies with Web service mechanisms to create a distributed system framework based around the Grid Service. Grid services conform to WSDL interfaces for such purpose as discovery of characteristics, notification, etc. OGSA introduces registration interfaces for creating and discovering services. The availability of lightpaths and cross connects is advertised through standards based registry services such as UDDI and WSIL (WSIL (Web Services Inspection Language). LPO (LightPath Object) registries contain all the services for creating the end-to-end lightpath. The LPO Factories are independent abstract components, which discover and register all provisioned end-to-end lightpaths for a given domain with dedicated Grid service registry. Network reachability information is exchanged between systems to construct the paths, allowing the creation and control of lightpaths. The solution allows communication with source and destination routes to create a new forwarding path along established lightpath and provides the capability to control the state of the lightpath. Interfaces have been developed to offer services for SAN application, which will query the lightpath registry services and establish an end-to-end lightpath. A policy manager is used to control user access. SAN needs can be translated in a policy condition. End-to-end lightpath are created according to a specified policy rule or defined network management policies. The policy server provides service for specifying and saving policies. The policy registry allows advertising the policy services. The signaling approach uses WSDL-based policy services for admission control and other policy services. The Grid resource management model is based on CIM (Common Information Model) [3] and its policy extension PCIM, allowing an efficient exchange of policy information. The preliminary implementation experiments demonstrate the Web-based signaling approach for creating and controlling the lightpath according to different policy conditions. The policy approach helps achieve an optimal utilization of network resources required by SAN applications.

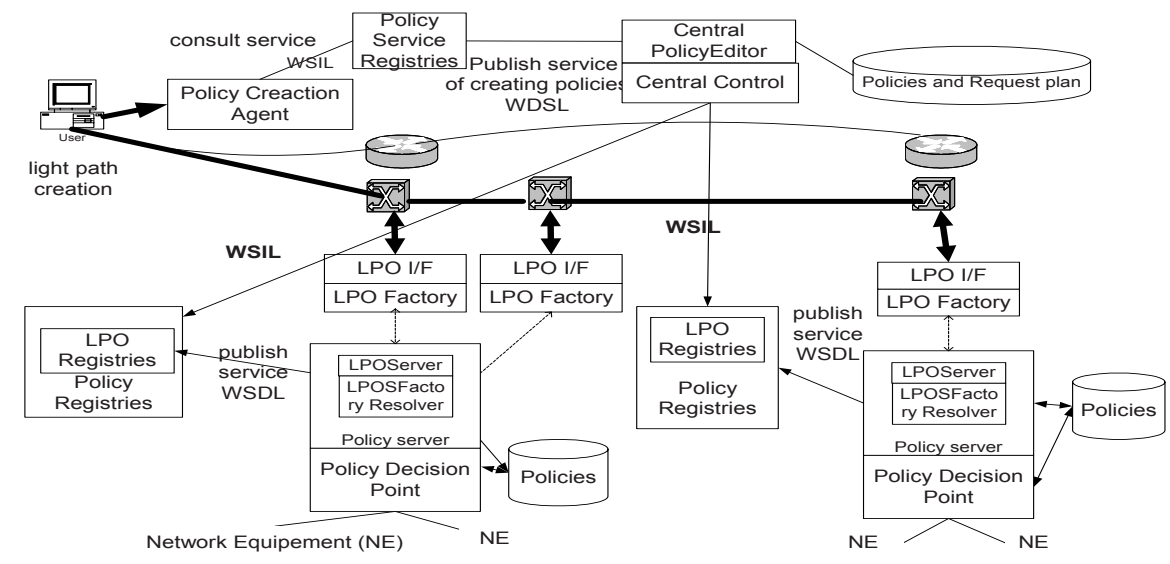

Fig. 1. Architecture 
We proposed a signaling approach based on the Open Grid architecture to dynamically provision lightpaths for end-to-end high volume SAN data transfers. SAN applications that need large high-speed bandwidths can use the policy services for controlling the access, set-up and tearing down the lightpaths based on policy conditions.

\section{References}

[1] Ravi Kumar Khattar et al, Introduction to Storage Area Network (SAN), Redbooks.

[2] I. Foster and al., "The Physiology of the Grid: An Open Grid Services Architecture for Distributed Systems Integration", OGSI WG, Global Grid Forum, June 22, 2002.

[3] CIM Schema, version 2.x. DMTF, http://www.dmtf.org/standards/standard_cim.php 